\title{
Lipid screening and treatment after acute coronary syndrome - the chasm between guidelines and real-life practice in a tertiary care center in Sri Lanka
}

\author{
Matthias $\mathrm{AT}^{1}$, Padmasiri MSM ${ }^{1}$ \\ Journal of the Ceylon College of Physicians, 2021, 52, 20-25
}

\begin{abstract}
Cardiovascular disease (CVD) remains the leading cause of death in Sri Lanka. Dyslipidaemia is a risk factor for CVD. Guidelines on dyslipidaemia recommends checking lipid levels in all patients admitted with acute coronary syndrome (ACS) and initiation of early high-intensity lipid-lowering therapy.
\end{abstract}

Patients admitted to the medical units of Colombo South Teaching Hospital were audited with two audit standards based on The National Institute of Health and Care Excellence (NICE) and European Society of Cardiology (ESC) guidelines.

Audit Standard one - All patients with ACS to have cholesterol measured within 24 hours of admission.

Audit Standard two - All patients to be given highintensity lipid-lowering therapy post ACS.

First audit cycle was during May-June 2020. Thereafter, the doctors were educated about the NICE and ESC guidelines and re-audited between July-September 2020 ( $2^{\text {nd }}$ cycle) closing the audit loop.

Both cycles had 200 patients each. In $1^{\text {st }}$ cycle, during the first 24 hours, 31 (15.5\%) patients had their total cholesterol (TC) and 2 (1\%) had a full lipid profile performed. During $2^{\text {nd }}$ cycle, 66 (33\%) patients had their TC and 11 (6.5\%) had a full lipid profile. Between the two cycles there was a statistically insignificant increase in lipid testing.
In 1 st cycle, high dose statins were prescribed in 199 (99.5\%) and in $2^{\text {nd }}$ cycle, 197 (98.5\%) patients. Hence high adherence to prescribing statins was seen. The lower number in the re-audit was due to comorbidities which prevented high-intensity statins.

Testing of lipids after ACS is poor and not keeping with above guideline recommendations. This study highlights the need for continuous education and motivation of doctors to improve adherence to guidelines on lipid screening in ACS. The usage of statins for secondary prevention is commendable.

Key words: lipid profile, statins, lipid screening, lowermiddle income country, acute coronary syndrome

\section{Introduction}

Cardio Vascular Disease (CVD) is the leading cause of death worldwide. In Sri Lanka, 34\% of mortality is due to CVD. ${ }^{1}$ South Asians develop coronary artery disease at an earlier stage in life. ${ }^{2} 77.4 \%$ of Sri Lankans have some form of dyslipidaemia. ${ }^{3}$ The link between blood lipid levels and coronary artery disease (CAD) risks was initially discovered decades ago. It is established that dyslipidaemia is a key risk factor for the development of atherosclerosis. To manage the CVD risk associated with dyslipidemia, identification and control of lipid markers is vital.

\footnotetext{
${ }^{1}$ Faculty of Medical Sciences, University of Sri Jayewardenepura, Nugegoda, Sri Lanka.
}

Correspondence: ATM, e-mail: thushara.matthias@sjp.ac.lk

https://orcid.org/0000-0003-0240-4845 
As evidence-based medicine has taken an important role in today's world, clinical practice guidelines as the primary mode of communicating current best practices for the management of dyslipidaemia and other non-communicable diseases. Current European Society of Cardiology (ESC) guidelines and NICE recommend that a full lipid profile is obtained for all patients presenting with an acute coronary syndrome (ACS) and repeated at three months. ${ }^{4,5}$ Lipid metabolism alters $24-48$ hours postmyocardial infarction, with a fall in total cholesterol (TC), LDL-C and high-density lipoprotein cholesterol (HDL-C), as well as a reciprocal increase in triglycerides (TG). ${ }^{6}$ The patient does not have to be fasting. ${ }^{7}$ It is recommended that cholesterol levels should be determined at admission as a baseline preinitiation of high-efficacy statin, and then reassessed after 4-6 weeks of therapy. If LDL goal is not achieved at this point, it is recommended to add ezetimibe to stain therapy. If the LDL goal is not achieved after adding stain and ezetimibe for 4-6 weeks, it is recommended to add $\mathrm{PCSK}^{9}$ inhibitors.

The 2019 ESC dyslipidemia guidelines emphasize the importance of initiating high-intensity lipid-lowering therapy early during hospital admission for patients with acute myocardial infarction (AMI) to reduce atherosclerotic risk by lowering LDL-C to levels. For patients at very high $\mathrm{CV}$ risk for secondary prevention, LDL-C reduction of $\geq 50 \%$ from baseline and a LDL-C goal of $<1.4 \mathrm{mmol} / \mathrm{L}(<55 \mathrm{mg} / \mathrm{dL}$ ) are recommended in the 2019 guidelines. ${ }^{4}$ It is recommended to give these patients high-intensity lipid-lowering therapy. This group of statins includes atorvastatin $40-80 \mathrm{mg}$, simvastatin $80 \mathrm{mg}$ and rosuvastatin 20-40 $\mathrm{mg}^{8}{ }^{8}$ In Sri Lanka, atorvastatin is the only statin available in the state sector hospitals. The few clinical scenarios where high dose statins will not be used are advanced age, hepatic or renal impairment and drug-interactions.

Numerous trials have proven the benefits of taking a higher dose of statin in reducing mortality. ${ }^{10}$ Therefore, high dose statins when compared to low dose satins have shown to be more effective in secondary prevention, thus, patients admitted with ACS are better discharged from the hospital on high dose statins as recommended by NICE and ESC guidelines. ${ }^{4}$

Despite the recommendation from international guidelines, clinical inertia is common in the management of dyslipidaemia. ${ }^{11,12,13}$ With the increased burden of CVD among South Asians, it was our aim to audit the lipid screening practices and adherence to prescribed statins for secondary prevention post myocardial infarction. We carried out an audit against two audit standards derived from the NICE and ESC guidelines.

\section{Method}

The audit was carried out at the Colombo South Teaching hospital (CSTH), one of the largest hospitals in Sri Lanka. It is a center that treats ACS patients in Colombo, the main commercial city and the largest city by population in Sri Lanka. CSTH is one of the leading teaching hospitals which provides treatment to about 150000 inward patients per year. It has a bed capacity of $1100 .{ }^{14}$ It has three medical units along with a specialized cardiology unit. It is also a teaching hospital that provides training to both undergraduate and postgraduate students. CSTH being a tertiary care hospital, is equipped with a full functional biochemistry lab with 24-hour laboratory cover.

Patients with chest pain, present to the emergency treatment unit and then are directed to the medical units after initial stabilization. If clinical, biochemical and ECG evidence suggest acute myocardial infarction, patients are treated according to the accepted protocols.

This was a single-site audit carried out over two separate, equal time periods during 2020 , with a view to improve our clinical practice against agreed internationally published standards. We carried out this audit against two audit standards derived from the NICE and ESC guidelines.

Standard One - all patients should undergo an assessment of serum cholesterol prior to discharge from hospital, preferably at 24 hours of admission.

Standard Two - all patients should be prescribed high-efficacy lipid-lowering therapy post-myocardial infarction for secondary prevention as tolerated, adjusting for significant comorbidities.

Patients admitted to medical units of CSTH between May to June 2020, and subsequently between mid-July to mid-September 2020 were recruited upon admission. Only patients fulfilling the criteria for a type 1 myocardial infarction were included. Patients aged 85 years and over were excluded. Clinical notes, biochemistry results and discharge summaries were reviewed during the ongoing admission, along with baseline demographics such as gender and age. Dates and details of cholesterol measurements were recorded. The drugs given on discharge were also noted. 
Education and awareness programs were delivered between audit cycles in order to improve the adherence to recommended clinical practice. Registrars, senior registrars and junior doctors including house officers were educated using a leaflet highlighting the recommendations from guidelines by means of face-to-face meetings. Following the initial awareness program, continuous reminders were given to doctors to ensure checking lipids post-ACS. Outcomes between audit cycles were compared using Fisher's exact test in SPSS (IBM SPSS Statistics Version 20 IBM Corp 2017. No patient identifiable data were stored.

\section{Results}

'Cycle one' was initially carried out to determine the compliance with current guidelines. After making number of interventions to improve our practice, 'Cycle two' was subsequently conducted to assess effect.

Cycle one: May to July 2020

200 patients were recruited during the cycle one. Of these, only 7 (3.5\%) patients had their Total cholesterol (TC) checked during admission, and 31 (15.5\%) patients had their TC checked within the first 24 hours of admission. A full lipid profile was ordered in 2 (1\%) patients on admission and in 7 (3.5\%) during first 24 hours, which comprised of TC, TG, HDL-C and LDL-C. There is a significant lack of adherence to audit standard. ${ }^{1}$ When discharge medications were reviewed, high dose statins were prescribed in 199 (99.5\%) patients for secondary prevention, demonstrating a very high adherence to standard two (Table 1).
Cycle two: July to September 2020

During this second two-month period, 200 patients with ACS were recruited. Regarding standard one, 18 (9\%) patients with ACS had their TC measured during their admission: 66 (33\%) patients had their TC checked in the first 24 hours of their admission. A full lipid profile was measured in just 2 patients on admission while 11 (6.5\%) patients had a lipid profile within first 24 hours. Disappointingly, 103 out of 200 (51.5\%) had no lipid screen of any kind, and 32 $(31.06 \%)$ of these patients were under 55 years of age, and $71(68.93 \%)$ of them were above 55 years of age. Of the patients who had their cholesterol measured in the first 24 hours of admission, the mean total cholesterol level was $180.48 \mathrm{mg} / \mathrm{dl}$ and mean LDL level was $126.47 \mathrm{mg} / \mathrm{dl}$. Maximum and minimum total cholesterol levels obtained were respectively 264.1 and 83.9. and $451 \mathrm{mg} / \mathrm{dl}$ respectively.

Following our intervention, there was a statistically insignificant increase in the total number of patients who had their cholesterol measured within 24 hours $(p=0.47)$. Furthermore, the increment in lipid profiles during cycle 2 was also not statistically significant $(p=1.000)$.

Regarding Standard Two and statin therapy, 197 (98.5\%) of patients were on appropriate secondary prevention, in accordance with the guidelines, at time of discharge. Of the 3 patients who were not discharged on statins, the cause was mentioned as due to renal failure in one case. No cause was mentioned in the other two cases. Given already good adherence in cycle one, this was a statistically insignificant reduction between cycles $(P<0.05)$.

Table 1. Audit standard adherence in the two cycles

\begin{tabular}{lll}
\hline Characteristic & Cycle 1 & Cycle 2 \\
\hline Number of participants & 200 & 200 \\
Total cholesterol checked during admission & $7(3.5 \%)$ & $18(9 \%)$ \\
Total cholesterol checked within 24 hours & $31(15.5 \%)$ & $66(33 \%)$ \\
Full lipid profile checked during admission & $2(1 \%)$ & $2(1 \%)$ \\
Full lipid profile checked within 24 hours & $7(3.5 \%)$ & $11(5.5 \%)$ \\
Discharged on high dose statins & $199(99.5 \%)$ & $197(98.5 \%)$ \\
\hline
\end{tabular}




\section{Discussion}

We audited the lipid screening practices for secondary prevention of patients with ACS at a large teaching hospital in Sri Lanka. The striking but clinically relevant disappointing finding of this audit was the poor adherence to guidelines in lipid testing after ACS. Despite advancements in dyslipidemia treatment and the existence of evidence-based standard care guidelines, many patients fail to achieve therapeutic targets in lipids in Sri Lanka. ${ }^{15}$ One of the reasons is "clinical inertia" in the management of dyslipidemia.

During our audit cycle one, we found that only $19 \%$ of our patient population had their total cholesterol checked in the first 24 hours of admission, falling far short of accepted international recommendations. The number of patients who had a complete lipid profile was low as $4.5 \%$, showcasing a drastic difference between the recommended ideal practice and the actual clinical practice. A similar audit done in UK at the Glasgow Royal Infirmary between January and May 2018 also showed poor adherence to guidelines. The TC checked in the first 24 hours of their admission was $61 \%$ and a full lipid profile was measured $36 \%$. Though still poor adherence to guidelines is seen, the figures are better than the present study. ${ }^{16}$ This of course shows that adherence to certain medical recommendations are poor even in developed countries and a frequent complaint of lack of financial resources may not be the actual reason behind not adhering to recommendations.

Following staff education, we demonstrated an increase in the number of ACS patients having their lipids measured wuthin the first 24 hours of admission, demonstrating a better compliance with standard one in cycle two of our clinical audit. But the increment between these two cycles were not statistically significant. Despite the observed improvement, the number of patients getting their cholesterol measured during admission for ACS remains poor and further improvements to practice is needed.

If we are to maintain the improvement seen in cycle 2 (the reaudit after educational activities), we need to identify the factors that result in poor adherence. There are organizational, systemic and provider factor involved in poor adherence to guidelines. High patient volumes and poor resources are potential problems at our center. On inquiring into the reasons for not doing lipid profile we found out that the doctors are not requesting the lipid profile assuming it is not done urgently. Usually in the ward setting, the serum sample is sent with a request form stating "blood for lipid profile" but proper clinical information as to why this sample is being sent is not clearly mentioned by the doctors requesting the sample. It is of utmost importance to indicate clearly that the sample being sent, belongs to a patient presenting with acute $\mathrm{Ml}$ and the importance of obtaining the lipid profile within 24 hours. This will enable the lab staff to carry out the lipid profile testing immediately and not give a future date for the testing to be done. There is a tendency for lab staff to give an elective date for fasting lipid profiles when such an indication is not clearly mentioned. On the other hand, a total cholesterol can be carried out easily in our lab setting. The reason why it was not done in the patients was due to doctors not requesting it. If the medical clerking becomes electronic this problem of writing the request forms properly will not occur and the dependence on the doctors to order the lipid profile can be overcome. The lipid profile can be included in the laboratory 'bundle' that is ordered when a patient is diagnosed as having ACS or presents with chest pain of cardiac origin. Sri Lanka is on its way to incorporating digital health into hospital information management systems and would incorporate electronic health records in the near future. Having electronic health records will mitigate the need to request individual investigations and lipid profile can be put as a bundle of investigations done for chest pain.

Although total cholesterol alone fulfills the basic requirement to comply with guidelines, neglecting a complete lipid profile will lead to several problems. If lipid profiles are not carried out common dyslipidemias such as low HDL and high TG which have been shown to be widely present in Sri Lankans would be missed. ${ }^{17}$ Pre statin lipid levels offer prognostic value as LDL levels can be optimally reduced to target levels if pre statin levels are known. The index cases of familial hypercholesterolemia can be diagnosed if only LDL values are known. If a full lipid profile is not done, we would not know the LDL value. This will yield to under diagnosing familial hypocholesteremia.

With regard to the providers, (doctors), lack of knowledge, insufficient awareness and familiarity with the guidelines is one of the main factors that result in poor adherence to guideline recommendation of carrying out lipid testing within first 24 hours. There is also poor knowledge about the applicability of the guidelines. In the absence of dyslipidemia guidelines of Sri Lanka, we need to use the best available evidence from other guidelines. This lack of awareness about guidelines affects practice. The Sri Lankan guidelines on dyslipidemia for secondary and tertiary care hospitals are in the process of being formulated at present. In clinical practice, the doctors do not request for TC nor lipid profile in patients with ACS. In poor resource countries, the providers constantly have a tendency to overestimate the care they provide. The 
providers assume that when high intensity statins are prescribed, it will invariably reduce the LDL to target levels. There is a significant proportion of patients who don't achieve the target LDL levels despite high dose statins. ${ }^{18,19,20}$ This has been shown in Sri Lanka as well as in other countries. If we are to offer non statin therapy to optimize the LDL levels, a pre statin full lipid profile is mandatory. This fact has to be educated to doctors to make them aware that merely prescribing high dose statins does not reduce CVD risk and that lipid profile need to be repeated to asses the response and if there is in adequate response, non statin therapy such as ezetimibe and $\mathrm{PCSK}^{9}$ inhibitors should be offered.

With regard to the second audit standard, our audit showed very good compliance. Atorvastatin is available in the state sector hospitals and prescribing it according to the guidelines is plausible in almost all state sector hospitals.

In order to improve guideline adherence, several strategies can be utilized. The method we used in this audit was education of the doctors. In order to educate the doctors about the guideline recommendations, we used a printed leaflet that was given to all doctors involved in the care of patients with ACS. We held face to face sessions educating them about guidelines and the facilities available in hospital for lipid testing. Other methods of educating doctors would be Continuous Medical Education (CME) programs. Audits such as the present audit will also help in creating awareness about guidelines. The results of this audit were made available to the staff. This encourages the doctors to change their practice and that bridges the science-gap. Further work is required for sustainable improvement in lipid profiling in our patients.

\section{Declarations}

Ethics approval and consent to participate: Ethical approval was obtained from the Ethics Review Committee of the Colombo South Teaching Hospital.

\section{Consent for publication}

Not applicable.

\section{Competing interests} interests.

The authors declare that they have no competing

\section{Availability of data and materials}

The datasets used during the current study are available from the corresponding author on reasonable request.

\section{Funding}

No Funding

\section{Authors' contributions}

ATM conceptualized the study and collected data, analyzed and drafted the paper. SP was involved in data collection and analysis and drafting.

\section{Acknowledgements}

Gayasha Somathilake, National Centre for Primary Care and Allergy Research, University of Sri Jayewardenepura, Sri Lanka for the input given on statistical analysis and lipid testing and treatment after acute myocardial infarction: no flags for the flagship by Louise Aubiniere-Robb, Jonathan E Dickerson, Adrian J B Brady published in Br J Cardiol 2019; 26: 141-4 for study design.

\section{References}

1. WHO | Noncommunicable diseases country profiles 2018 . WHO. 2018.

2. Joshi P, Islam S, Pais P, et al. Risk Factors for Early Myocardial Infarction in South Asians Compared With Individuals in Other Countries. JAMA 2007; 297(3): 286. doi:10.1001/jama.297.3.286

3. Katulanda P, Dissanayake HA, De Silva SDN, et al. Prevalence, patterns, and associations of dyslipidemia among Sri Lankan adults - Sri Lanka Diabetes and Cardiovascular Study in 2005-2006. J Clin Lipidol. 2018; 12(2): 447-54. doi:10.1016/j.jacl.2018.01.006

4. 2019 ESC/EAS Guidelines for the management of dyslipidaemias: lipid modification to reduce cardiovascular risk. doi:10.1093/eurheartj/ehz455

5. Lipid modification: Cardiovascular risk assessment and the modification of blood lipids for the primary and secondary prevention of cardiovascular disease | Guidance | NICE.

6. Pitt B, Loscalzo J, Ycas J, Raichlen JS. Lipid Levels After Acute Coronary Syndromes. J Am Coll Cardiol. 2008; 51(15): 1440-5. doi:10.1016/j.jacc.2007.11.075

7. Chapman MJ, Ginsberg HN, Amarenco P, et al. Triglyceriderich lipoproteins and high-density lipoprotein cholesterol in patients at high risk of cardiovascular disease: Evidence and guidance for management. Eur Heart J. 2011; 32(11): 1345-61. doi:10.1093/eurheartj/ehr112

8. 2018 AHA/ACC Multisociety Guideline on the Management of Blood Cholesterol - American College of Cardiology. https://www.acc.org/latest-in-cardiology/ten-points-toremember/2018/11/09/14/28/2018-guideline-onmanagement-of-blood-cholesterol. Accessed November 27, 2019. 
9. Stancu C, Sima A. Statins: Mechanism of action and effects. J Cell Mol Med. 2001; 5(4): 378-87. doi:10.1111/ j.1582-4934.2001.tb00172.x

10. An K, Huang R, Tian S, et al. Statins significantly reduce mortality in patients receiving clopidogrel without affecting platelet activation and aggregation: A systematic review and meta-analysis. Lipids Health Dis. 2019; 18(1): 1-11. doi:10.1186/s12944-019-1053-0

11. Phillips LS, Branch J, Cook CB, et al. Clinical inertia. Ann Intern Med. 2001; 135(9): 825-834. doi:10.7326/00034819-135-9-200111060-00012

12. Pittman DG, Fenton C, Chen W, Haffner S, Pendergrass M. Relation of statin nonadherence and treatment intensification. Am J Cardiol. 2012; 110(10): 1459-63. doi:10.1016/j.amjcard.2012.07.008

13. Rodondi N, Peng T, Karter AJ, et al. Therapy modifications in response to poorly controlled hypertension, dyslipidemia, and diabetes mellitus. Ann Intern Med. 2006; 144(7): 475-84. doi:10.7326/0003-4819-144-7200604040-00006

14. Https://www.csth.health.gov.Ik/about-us. Inpatient. MOH.

15. Do we achieve LDL-cholesterol targets in routine clinical practice? Evidence from a tertiary care hospital in Sri Lanka | Wijekoon | Proceedings of Annual Scientific
Sessions of Faculty of Medical Sciences. http:// journals.sjp.ac.Ik/index.php/ASS/article/view/4181. Accessed April 14, 2020.

16. Louise Aubiniere-Robb, Jonathan E Dickerson, Adrian J B Brady, Lipid testing and treatment after acute myocardial infarction: no flags for the flagship. Br J Cardiol. 2019. doi:10.5837/bjc.2019.041

17. Katulanda P, Dissanayake HA, De Silva SDN, et al. Prevalence, patterns, and associations of dyslipidemia among Sri Lankan adults - Sri Lanka Diabetes and Cardiovascular Study in 2005-2006. J Clin Lipidol. 2018; 12(2): 447-54. doi:10.1016/j.jacl.2018.01.006

18. Do we achieve LDL-cholesterol targets in routine clinical practice? Evidence from a tertiary care hospital in Sri Lanka | Wijekoon | Proceedings of Annual Scientific Sessions of Faculty of Medical Sciences.

19. Pittman DG, Fenton C, Chen W, Haffner S, Pendergrass M. Relation of statin nonadherence and treatment intensification. Am J Cardiol. 2012; 110(10): 1459-63. doi:10.1016/j.amjcard.2012.07.008

20. Rodondi N, Peng T, Karter AJ, et al. Therapy modifications in response to poorly controlled hypertension, dyslipidemia, and diabetes mellitus. Ann Intern Med. 2006; 144(7): 475-484. doi:10.7326/0003-4819-144-7200604040-00006 